\title{
Métodos baseados em Atlas ou TW3? Estimativa da idade óssea em brasileiros
}

\author{
Atlasbased methods or TW3?- Estimation of bone age in Brazilians
}

\author{
Ana I. Ortega-Pertuz ${ }^{1 *}$, Francisco Haiter-Neto ${ }^{2}$, Andre L. Ferreira Costa ${ }^{3}$
}

\begin{abstract}
RESUMO
Objetivo: Comparar os métodos de Greulich-Pyle (GP), o do Atlas Venezuelano de Maturação Óssea (AV) e o de Tanner-Whitehouse (TW3-RUS), na estimativa da idade em brasileiros. Metodologia: Utilizou-se uma amostra de 240 radiografias carpais de indivíduos de ambos os sexos (129 meninas e 111 meninos), com idades entre 84 e 199 meses (7 e 16,5 anos). As idades ósseas foram calculadas segundo os métodos GP, AV e TW3-RUS e posteriormente se obtiveram diferenças de media entre a idade cronológica (IC) as idades ósseas estimadas. Resultados: Observou-se uma correlação positiva e estatisticamente significativa entre a IC e as idades ósseas estimadas, $(0,89-0,94)$. Constatou-se uma superestimativa da idade por todos os métodos nas meninas. Nos meninos verificou-se a superestimativa pelo método AV, enquanto que o GP e o TW3-RUS subestimaram a idade. Verificou-se uma diferença significativa entre a IC e as idades ósseas estimadas pelos métodos GP e AV em ambos os sexos (meninas: GP -6,89; AV -6,41. Meninos: GP 4,45; AV-5,06) e para o TW3-RUS somente foi significativa para as meninas (meninas -2,94; meninos 1,56). Conclusões: O método TW3-RUS resultou ser mais preciso na estimativa da idade, porém se recomenda a utilização das equações de regressão calculadas para um melhor ajuste entre as idades inferidas pelos métodos estudados as idades reais dos indivíduos.
\end{abstract}

Palavras-chave: Crescimento e Desenvolvimento. Determinação da Idade pelo Esqueleto. Atlas de Greulich-Pyle. Método de Tanner-Whitehouse. Atlas Venezolano de Maduración Ósea.

\begin{abstract}
Objective: To compare the methods of Greulich-Pyle (GP), the Venezuelan Atlas of Bone Maturation (VA), and Tanner-Whitehouse (TW3-RUS), for age estimation in Brazilians. Methodology: A sample of 240 carpal radiographs from individuals of both sexes (129 females and 111 males), with chronological ages (CA) between 84 to 199 months (7 - 16.5 years old) was used. Bone ages were calculated using GP, VA and TW3-RUS methods. Mean differences between CA and estimated bone ages were calculated.
\end{abstract}

1. Doutora, Professora Titular. Área de Odontologia Forense. Instituto de Investigaciones. Facultad de Odontología. Universidad del Zulia (LUZ). Maracaibo, Estado Zulia, Venezuela.

2. Doutor, Professor Titular. Área de Radiologia Odontológica. Departamento de Diagnóstico Oral. Faculdade de Odontologia de Piracicaba, Universidade Estadual de Campinas (UNICAMP). SP, Brasil.

3. Doutor, Professor Associado. Departamento de Ortodontia. Universidade Cidade de São Paulo (UNICID), São Paulo, SP, Brasil.
Correspondência: Dra. Ana Isabel Ortega. Laboratorio de Odontología Forense. Área de Odontología Forense, Instituto de Investigaciones. Facultad de Odontología. Universidad del Zulia (LUZ). Calle 65 con Avenida 19. Maracaibo, Estado Zulia. Venezuela. anitaortegav@gmail.com.

Artigo recebido em 02/12/2013 Aprovado para publicação em 19/08/2014 
Results: There was a positive and statistically significant correlation between CA and bone ages (0.89 0.94). An age overestimation was observed by all methods in girls. In boys, overestimation was observed using the VA, while GP and TW3-RUS underestimated age. There was a significant difference between CA and bone ages estimated by GP and VA in both sexes (girls: GP -6.89, -6.41 AV; boys: GP 4.45; VA-5, 06). For TW3-RUS it was only significant for girls (-2.94 girls; boys 1.56). Conclusions: TW3-RUS method resulted in more accurate age estimation. It is recommended to use the calculated regression equations for a better fit between inferred ages through the studied methods and the individual's real ages.

Key words: Growth and Development. Age Determination by Skeleton. Greulich-Pyle Atlas. TannerWhitehouse method. Venezuelan Atlas of Bone Maturation.

\section{Introdução}

A idade óssea (IO) é o indicador da idade biológica mais útil para caracterizar ritmos de maturação e tem demonstrado ser mais preciso e confiável que a idade cronológica (IC) na avaliação do progresso do individuo para a maturidade. É influenciada pelo sexo, grupo étnico e fatores genéticos, nutricionais, ambientais e socioeconômicos. Como toda informação relacionada com o crescimento e desenvolvimento, a IO deve ser referida a padrões de normalidade próprios da coletividade à qual pertence o individuo em estudo. ${ }^{1,2}$

A determinação da IO tem um papel importante na pediatria, especialmente no diagnóstico e seguimento de crianças com transtornos de crescimento relacionados com desordens endócrinas, metabólicas, genéticas, ortopédicas ou renais assim como na predição da estatura adulta. ${ }^{2} \mathrm{Na}$ prática forense, a IO incluí-se dentro do exame integral para o diagnóstico da idade acompanhada do registro de dados antropométricos, sinais de maturação sexual, desordens de desenvolvimento relevantes para a estimativa da idade, um exame dental que recolhe informação sobre o status da dentição e uma radiografia panorâmica para a estimativa da idade dental. ${ }^{3}$ A IO é estimada na radiografia carpal, quando se trata de indivíduos em desenvolvimento e em sujeitos com idades ao redor de 18 anos se recomenda uma radiografia ou tomografia computadorizada da clavícula. ${ }^{3,4}$

Encontram-se diversos métodos para estimar a IO em radiografias carpais. Alguns utilizam um atlas, como o de Greulich-Pyle ${ }^{5}$ (GP) ou de Maduração Óssea do Venezuelano ${ }^{6}(\mathrm{AV})$. Este último foi padronizado com uma amostra de 4.200 crianças venezuelanas de ambos os sexos, pertencentes a todos os estratos sociais. ${ }^{6}$ Os atlas consistem numa coleção de imagens de radiografias selecionadas como protótipo o padrão, nos quais os ossos mostrados apresentam um estágio de maduração típico para una IC específica. A aplicação do método implica comparar a radiografia em estudo com as imagens do atlas, com a finalidade de encontrar aquela que melhor represente o grau de desenvolvimento do individuo. A idade associada à radiografia "padrão" do atlas representa a IO., ${ }^{5,6}$ Outros métodos designam escores a centros de ossificação específicos de acordo a estágios de maturação. Tais escores são somados para obter um índice de maturação esquelética que posteriormente é transformado em IO, mediante tabelas específicas por sexo, entre eles o mais empregado é o de Tanner-Whitehouse (TW). ${ }^{1}$

Alguns autores têm investigado a aplicabilidade dos métodos de GP e TW em indivíduos com características genéticas, étnicas, socioeconômicas e ambientais diferentes da amostra empregada para padronizá-los. ${ }^{7-16}$ Vários trabalhos tem demonstrado uma subestimativa da IO em relação à idade cronológica quando se utiliza o método de GP, tal como foi constatado em australianos ${ }^{7}$, brasileiros ${ }^{8-10}$, finlandeses $^{11}$, indianos ${ }^{12}$, italianos ${ }^{13}$, em indivíduos de Europa central ${ }^{14}$, malawenses ${ }^{15}$ e turcos. ${ }^{16}$ Quando se emprega o método TW, as idades ósseas estimadas são muitas vezes maiores que a IC, o que tem sido observado em argentinos ${ }^{17}$, italianos ${ }^{18}$ e japoneses. ${ }^{19} \mathrm{Em}$ relação ao $\mathrm{AV}$, se desconhece se o mesmo tem sido aplicado em outras populações para verificar sua aplicabilidade.

Considerando que a utilidade de um método de estimativa da IO pode ver-se comprometida devido a diferenças entre a população empregada para a padronização do método e o individuo em estudo, assim como por fatores inerentes à utilização prática do mesmo, este trabalho se propôs avaliar a aplicabilidade dos métodos de GP, TW o AV na estimativa da idade, numa amostra brasileira. 


\section{Material e métodos}

A amostra consistiu em 240 radiografias carpais, selecionadas de forma intencional e não probabilística, do arquivo da Disciplina de Radiologia da Faculdade de Odontologia de Piracicaba da UNICAMP, pertencentes a indivíduos brasileiros saudáveis de acordo com a ficha clínica que acompanhava cada radiografia, de ambos os sexos (129 meninas e 111 meninos), com idades cronológicas entre 84 e 199 meses, residentes na cidade de Piracicaba, estado de São Paulo, Brasil. As radiografias foram agrupadas em nove faixas etárias, com uma diferença de 11 meses entre cada uma, constituídas por 10 indivíduos como mínimo. ${ }^{20} \mathrm{O}$ estudo foi aprovado pelo Comitê de Ética local (parecer 037/2002).

Para a seleção das radiografias foram considerados os seguintes critérios: (a) achados normais em tecidos moles e ósseos na imagem radiográfica, (b) sem evidência clínica, de acordo a ficha clínica, de transtornos de crescimento o desenvolvimento, (c) adequada projeção radiográfica dos centros de ossificação em avaliação, (d) densidade e contraste adequados.

As idades ósseas foram estimadas por meio dos métodos de GP, AV e TW, este último com seu sistema RUS (rádio, ulna e ossos curtos) da versão TW3 do método. As estimativas foram realizadas em duas fases. A primeira consistiu na aplicação dos métodos de GP e TW3-RUS nas radiografias da amostra, por cinco observadores devidamente treinados. Por razões de distância geográfica, um dos autores (AIO) aplicou o AV nas imagens digitalizadas das radiografias originais, obtidas mediante um escâner (HP scanjet 4C series, Hewlett Packard Company, Palo Alto, California, USA) com resolução de 300 dpi e armazenadas no formato JPG. As estimativas da IO nas imagens digitais se realizaram num monitor de tela plana de 20 polegadas, utilizando o software Adobe Photoshop, versão 12.0 (Adobe Systems, Inc, San José, CA). O observador empregou os recursos de brilho, contraste e magnificação do software. Quando se utiliza o AV, o avaliador seleciona entre as imagens padrão do atlas, a que mais se assemelha a radiografia em estudo, de forma similar a aplicação do GP.

Os avaliadores só tinham conhecimento do sexo do paciente e determinaram as idades ósseas duas vezes por cada método, com um intervalo de 15 dias entre uma e outra avaliação, com o objetivo de evitar a influencia da memorização das radiografias sobre o segundo exame. Todas as idades ósseas foram transformadas em meses para posterior análise estatística.

Para a análise dos dados utilizou-se o programa estatístico SPSS versão 15 para Windows (SPSS Inc. ${ }^{\circledR}$, Chicago, IL, USA). A calibração dos observadores realizou-se através da determinação das diferencias intra e interobservador mediante uma análise de variância. A tabulação da informação realizou-se quando não se encontraram diferenças estatisticamente significativas entre os valores. Obtiveram-se médias, desvios-padrão e o erro padrão da média para a IC e as idades ósseas estimadas, assim como os coeficientes de correlação de Pearson entre estas variáveis. Ainda, aplicou-se um teste de diferença de média entre a IC e as idades ósseas calculadas através dos diferentes métodos. Também se realizou uma análise de regressão simples utilizando a IC como variável dependente, para obter modelos que permitiram um melhor ajuste das idades ósseas estimadas e as idades cronológicas dos indivíduos estudados. O nível de significância assumido foi de $p \leq 0,05$.

\section{Resultados}

A análise de correlação de Pearson entre a IC e as idades ósseas estimadas através dos métodos estudados mostrou una correlação altamente positiva e significativa entre as variáveis (Meninas: GP 0,94; AV 0,94; TW3-RUS 0,89. Meninos: GP 0,91; AV 0,90; TW3-RUS 0,92). Nas Tabelas 1 e 2 apresentam-se as médias da IC e as médias das idades ósseas determinadas através dos métodos, para cada faixa etária. No sexo feminino (Tabela 1) constatou-se que as médias das idades ósseas calculadas por GP foram maiores que a IC em todos os grupos de idade, com exceção do segundo grupo. Para o AV as médias da EO foram maiores que as médias da IC na maioria dos grupos estudados. As médias das idades ósseas calculadas pelo TW3-RUS foram superiores as medias da IC nos terceiro ao oitavo grupos.

Na Tabela 2 observa-se no sexo masculino, que as médias das idades ósseas estimadas pelo método de GP foram menores que a média da IC do primeiro ao quarto grupo e nos grupos de idade seis e nove. Para o AV as idades ósseas estimadas foram maiores que as médias da IC desde o terceiro grupo de idade. Por outro lado, o sistema TW3-RUS mostrou uma subestimação da idade do primeiro ao quarto grupo e no nono grupo. Estes resultados são ilustrados nas Figuras 1 e 2, onde se observam as correlações entre 


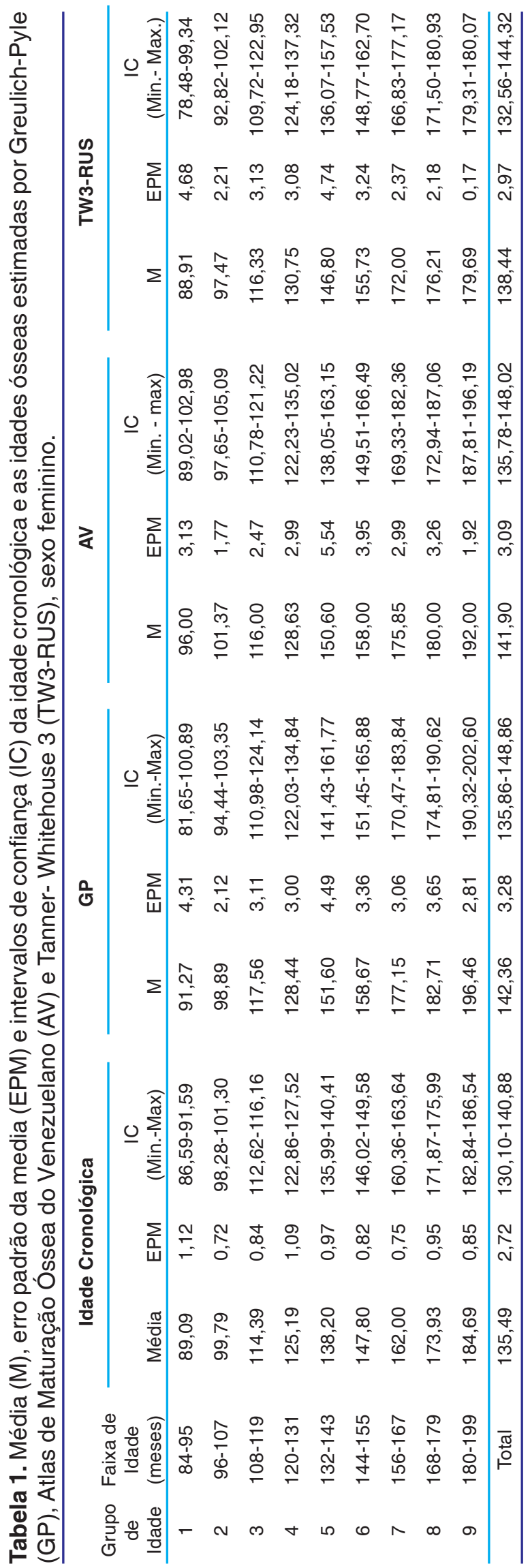

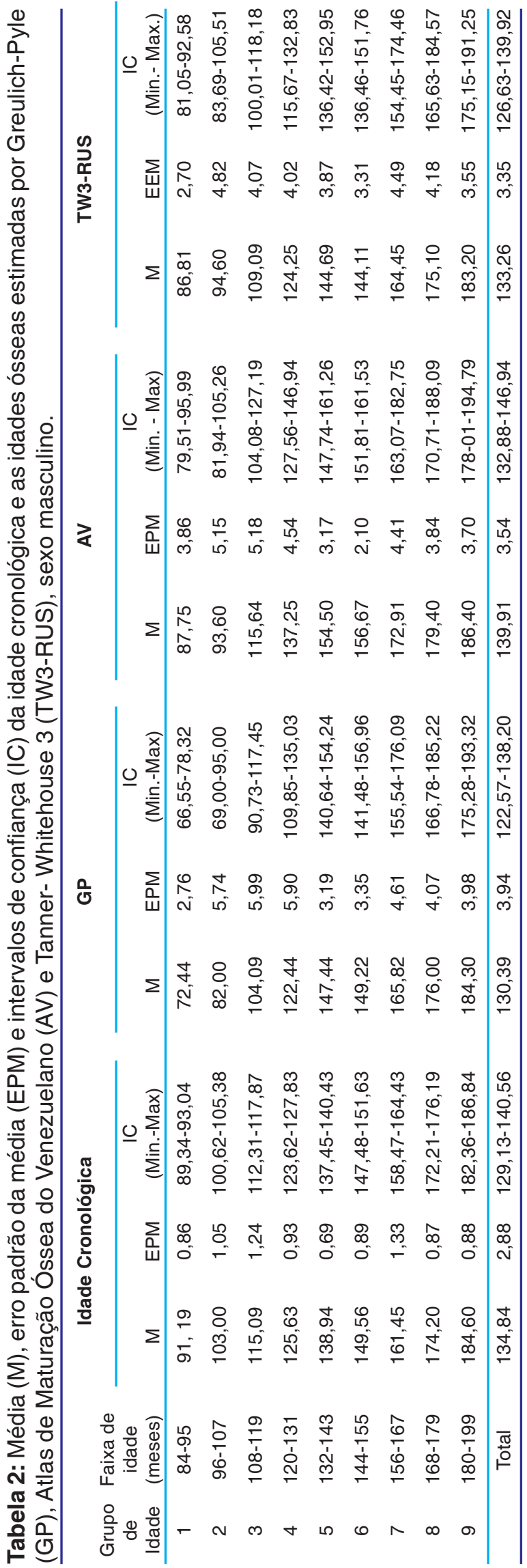




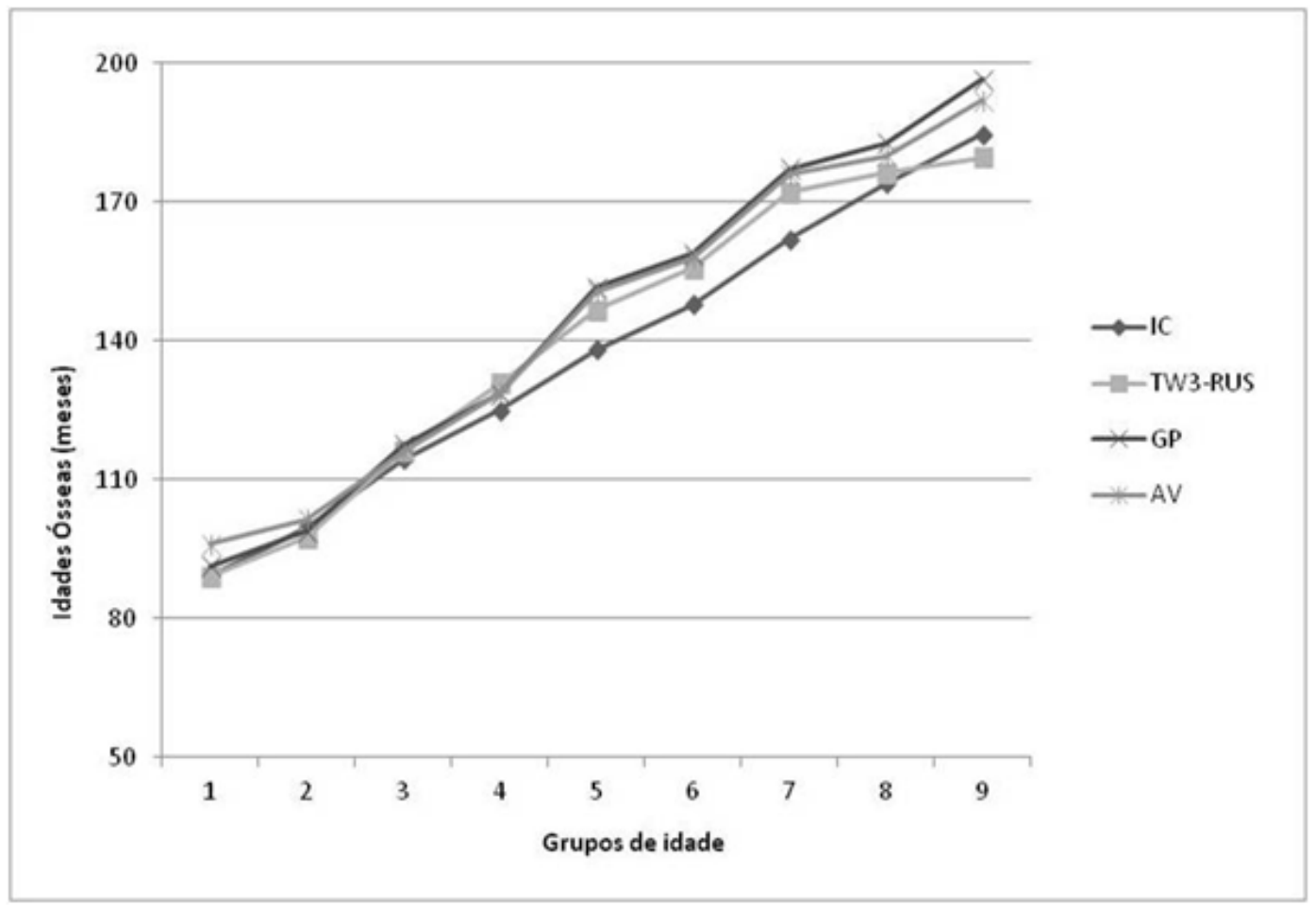

Figura 1. Correlações entre a idade cronológica (IC) e as idades estimadas pelos métodos de Greulich-Pyle (GP), Atlas Venezuelano de Maturação Óssea (AV) e Tanner-Whitehouse (TW3-RUS). Sexo feminino.

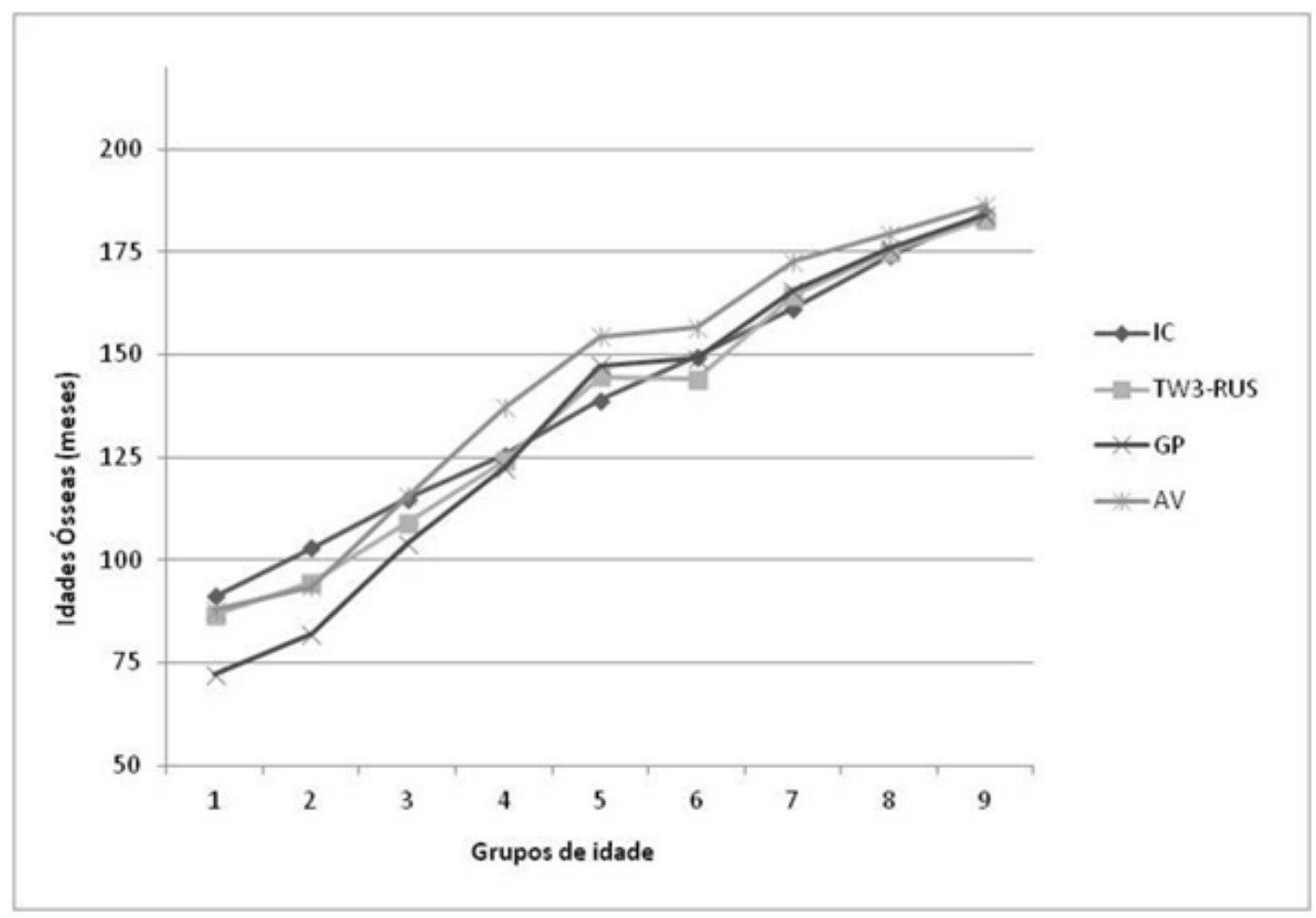

Figura 2. Correlações entre a idade cronológica (IC) e as idades estimadas pelos métodos de Greulich-Pyle (GP), Atlas Venezuelano de Maturação Óssea (AV) e Tanner-Whitehouse (TW3-RUS). Sexo masculino. 
as idades ósseas estimadas e a IC, em meninas e meninos, respectivamente. Nos meninos, se observou a subestimação da idade nos grupos mais jovens, em tanto que nas meninas se verificou uma consistente superestimativa por todos os métodos.

Observou-se uma diferença estatisticamente significativa entre a IO e as idades estimadas por GP e AV (considerando o total da diferença) em ambos os sexos. O TW3-RUS mostrou a menor diferença com respeito à IC (Tabela 3). Nas meninas observouse que para o GP houve uma diferença estatisticamente significativa entre a IC e a IO estimada pelo método em todos os grupos de idade, para o AV e TW3-RUS esta diferença foi constatada nos grupos seis, sétimo e nono. Nos meninos, o GP mostrou uma diferença significativa no primeiro, segundo, quinto e sexto grupos de idade, enquanto que no AV a diferença foi verificada nos grupos quinto ao sétimo. Para o TW3RUS não se observou uma diferença significativa entre as variáveis em todas as idades estudadas.

A Tabela 4 apresenta os modelos de regressão construídos por meio das idades ósseas estimadas por GP, AV e TW3-RUS, para ambos os sexos, os mesmos devem ser empregados para ajustar os métodos à amostra estudada.

\section{Discussão}

A IO expressa com grande fidelidade o grau de desenvolvimento do individuo, por isto é utilizada para o diagnóstico de transtornos de crescimento. ${ }^{2}$ Assim, a associação entre a IC e a IO tem permitido que esta última fosse empregada com sucesso na estimativa da idade dentro dos processos de identificação médico-legais realizados em sujeitos vivos ou falecidos. ${ }^{4}$

Tabela 3: Diferença média ( \pm desvío padrão) entre a idade cronológica (IC) e as idades estimadas pelos métodos Greulich- Pyle (GP), Atlas Venezuelano de Maturação Óssea (AV) e Tanner-Whitehouse (TW3-RUS).

\begin{tabular}{|c|c|c|c|c|c|c|c|}
\hline \multirow{3}{*}{$\begin{array}{c}\begin{array}{c}\text { Grupo } \\
\text { de idade }\end{array} \\
1\end{array}$} & \multirow[t]{2}{*}{$\begin{array}{l}\text { Faixa de } \\
\text { idade } \\
\text { (meses) }\end{array}$} & & & \multicolumn{2}{|c|}{ IC-AV } & \multicolumn{2}{|c|}{ IC-TW3RUS } \\
\hline & & \multicolumn{6}{|c|}{ Sexo Feminino } \\
\hline & $84-95$ & $-2,18$ & $\pm 15,21$ & $-6,90$ & $\pm 10,83$ & 0,18 & $\pm 16,43$ \\
\hline 2 & $96-107$ & 0,89 & $\pm 10,38$ & $-1,57$ & $\pm 8,96$ & 2,31 & $\pm 10,61$ \\
\hline 3 & $108-119$ & $-3,16$ & $\pm 12,66$ & $-1,61$ & $\pm 10,53$ & $-1,94$ & $\pm 13,18$ \\
\hline 4 & $120-131$ & $-3,25$ & $\pm 12,86$ & $-3,43$ & $\pm 13,68$ & $-5,56$ & $\pm 13,87$ \\
\hline 5 & $132-143$ & $-13,40^{\star}$ & $\pm 15,01$ & $-12,40$ & $\pm 17,16$ & $-8,60$ & $\pm 14,53$ \\
\hline 6 & $144-155$ & $-10,86^{*}$ & $\pm 12,21$ & $-10,20^{*}$ & $\pm 14,52$ & $-7,93^{\star}$ & $\pm 11,52$ \\
\hline 7 & $156-167$ & $-15,15^{\star}$ & $\pm 8,96$ & $-13,84^{*}$ & $\pm 8,86$ & $-10,00^{\star}$ & $\pm 6,73$ \\
\hline 8 & $168-179$ & $-8,78^{*}$ & $\pm 13,89$ & $-6,07$ & $\pm 12,21$ & $-2,28$ & $\pm 8,48$ \\
\hline 9 & $180-199$ & $-11,76^{\star}$ & $\pm 10,57$ & $-7,30^{*}$ & $\pm 8,08$ & $5,00^{*}$ & $\pm 2,97$ \\
\hline \multirow[t]{2}{*}{ Total } & & $-6,86^{*}$ & $\pm 13,15$ & $-6,41^{*}$ & $\pm 12,15$ & $-2,94$ & $\pm 12,13$ \\
\hline & & \multicolumn{6}{|c|}{ Sexo Masculino } \\
\hline 1 & $84-95$ & $18,75^{\star}$ & $\pm 9,78$ & 3,43 & $\pm 14,45$ & 4,37 & $\pm 9,93$ \\
\hline 2 & $96-107$ & $21,00^{*}$ & $\pm 19,70$ & 9,40 & $\pm 17,75$ & 8,40 & $\pm 16,66$ \\
\hline 3 & $108-119$ & 11,00 & $\pm 20,18$ & $-0,54$ & $\pm 17,25$ & 6,00 & $\pm 14,14$ \\
\hline 4 & $120-131$ & 3,18 & $\pm 22,43$ & $-11,62$ & $\pm 16,91$ & 1,37 & $\pm 14,88$ \\
\hline 5 & $132-143$ & $-8,50^{\star}$ & $\pm 11,95$ & $-15,56^{\star}$ & $\pm 11,79$ & $-5,75$ & $\pm 14,64$ \\
\hline 6 & $144-155$ & 0,33 & $\pm 11,65$ & $-7,11^{*}$ & $\pm 7,84$ & 5,44 & $\pm 11,05$ \\
\hline 7 & $156-167$ & $-4,36^{*}$ & $\pm 15,56$ & $-11,45^{\star}$ & $\pm 14,45$ & $-3,00$ & $\pm 14,92$ \\
\hline 8 & $168-179$ & $-1,80$ & $\pm 14,18$ & $-5,20$ & $\pm 13,82$ & $-0,90$ & $\pm 14,19$ \\
\hline 9 & $180-199$ & 0,30 & $\pm 12,63$ & $-1,90$ & $\pm 11,96$ & 1,40 & $\pm 11,07$ \\
\hline Total & & $4,45^{\star}$ & $\pm 18,31$ & $-5,06^{*}$ & $\pm 15,92$ & 1,56 & $\pm 13,85$ \\
\hline
\end{tabular}

* Estatisticamente significante. 
Tabela 4: Modelos de regressão simples com a idade cronológica (IC) como variável dependente, calculada por médio das idades ósseas estimadas pelo Greulich-Pyle (GP), Atlas de Maturação Óssea do Venezuelano (AV) e Tanner- Whitehouse (TW3-RUS).

\begin{tabular}{lccccc}
\hline Sexo & Modelo & Constante & Beta & Significância & $\mathbf{R}^{2}$ \\
\hline Feminino & GP & 24,10 & 0,78 & $0,000^{*}$ & 0,88 \\
& AV & 17,84 & 0,82 & $0,000^{*}$ & 0,88 \\
& TW3-RUS & 16,94 & 0,85 & $0,000^{*}$ & 0,87 \\
Masculino & & & & \\
& GP & 47,79 & 0,66 & $0,000^{*}$ & 0,83 \\
& AV & 31,64 & 0,73 & $0,000^{*}$ & 0,82 \\
& TW3-RUS & 29,48 & 0,79 & $0,000^{*}$ & 0,79 \\
\hline
\end{tabular}

* Estatisticamente significante.

Nesta pesquisa, se demonstrou uma forte associação entre a IC e as idades ósseas estimadas, encontrando-se em ambos os sexos, coeficientes de correlação altos para todos os métodos, em concordância com diversos trabalhos que avaliaram a aplicabilidade de GP e TW em amostras brasileiras. ${ }^{8,} 10,21,22$ Estudos têm observado diferenças entre a IC e a IO calculada, sugerindo que as mesmas se produzem pelas variações no ritmo e velocidade de maturação esquelética da população em estudo com a utilizada na padronização do mesmo. ${ }^{12,23-26}$

Conforme os resultados, observou-se que as idades ósseas estimadas pelo método de GP foram adiantadas com respeito à IC no sexo feminino, para o sexo masculino se constatou o atraso da IO, esta variação entre os sexos também foi reportada por Haiter-Neto et $\mathrm{al}^{10}$ (2000), Amaral et $\mathrm{al}^{21}$ (2002) e Cantekin et al ${ }^{26}$ (2012). Em contraste, outros autores têm encontrado uma subestimativa da idade para ambos os sexos. ${ }^{12,13,15,16,27} \mathrm{O}$ AV superestimou as idades para meninas e meninos, sendo esta superestimativa maior para o sexo feminino. Para o TW3-RUS se constatou uma superestimação nas meninas e uma subestimativa para o masculino, porém é importante salientar que as idades ósseas foram próximas a IC, como foi encontrado Vignolo et $\mathrm{al}^{18}$ (1990), HaiterNeto et $\mathrm{al}^{28}$ (2006) e Buken et $\mathrm{al}^{25}$ (2010) quando utilizaram esta versão do método TW.

Em relação às diferenças (totais) entre a IC e as idades ósseas estimadas, estas foram estatisticamente significativas para os métodos baseados em atlas. Nas meninas as diferenças foram maiores durante a puberdade para GP e $\mathrm{AV}$, o que foi somente observado nos meninos para o $\mathrm{AV}$, enquanto que com o GP os indivíduos do sexo masculino apresentaram as maiores diferenças nos grupos de idades mais jovens. $\mathrm{O}$ avance da $\mathrm{IO}$ com respeito à IC na puberdade pode estar associado com o surto de crescimento, o qual foi mais evidente nas meninas. $\mathrm{O}$ valor da diferença média de IC-IO estimada por GP foi superior ao reportado por Loder et $\mathrm{al}^{29}$ (1993) e Zhang et $\mathrm{al}^{23}$ (2009), e inferior ao verificado por Lewis et al $^{15}$ (2002) e Calfe et $\mathrm{al}^{24}$ (2010).

No entanto, o TW3-RUS mostrou a menor diferença (total) dos três métodos estudados, com diferenças menores de um ano em todos os grupos de idade, para ambos os sexos. Os valores obtidos neste trabalho foram inferiores aos observados por Vignolo et $\mathrm{al}^{18}$ (1990) e superiores aos resultados de Buken et $\mathrm{al}^{25}$ (2010), mas próximos com as diferenças mostradas por Schmidt et $\mathrm{al}^{30}$ (2008).

Mesmo observando diferenças totais menores de 12 meses para todos os métodos a presença de grupos de idade com resultados de até 21 meses e desvios padrão com uma magnitude de 22,43 meses nos métodos baseados em atlas, faz com que os dados da estimativa de idade obtidos por médio de GP e AV, devam ser interpretados com cautela. Por isto, a inclusão de informação de idade dental, estatura, peso, a presença e grau de desenvolvimento dos caracteres sexuais secundários é imperativa nos processos de determinação da idade, especialmente quando os métodos disponíveis para a avaliação não são próprios da população em estudo.

Em relação à seleção do método de estimativa de idade, os resultados indicaram que o TW3-RUS fornece idades mais próximas à idade real dos indivíduos. Além de considerar a possibilidade de que a 
maturação dos indivíduos estudados fosse mais próxima a dos sujeitos selecionados para a padronização do método, cabe salientar o componente de subjetividade nas avaliações feitas utilizando ambos os atlas, desde que o enfoque empregado neste estudo foi a comparação geral da radiografia com uma imagem, e não uma média das idades dos centros de ossificação.

Uma limitação desta pesquisa foi a não inclusão de sujeitos com idades superiores aos dezesseis anos, o que restringe realizar inferências relacionadas com aplicabilidade dos métodos em sujeitos próximos a maioridade, onde é critica a utilização de métodos que superestimam a idade, podendo incorrer em erros técnicos, éticos e jurídicos inaceitáveis, classificando um individuo menor de idade como adulto, o que é importante na pratica forense. As equações de regressão aqui apresentadas, permitiram ajustar os métodos à amostra em estudo para o calculo da idade, porém há necessidade de desenvolver referencias específicas para a população brasileira.

\section{Conclusão}

Com base nos resultados obtidos nos processos de estimação usando os métodos de GP, AV e TW3RUS, observou-se correlações positivas entre as idades ósseas calculadas e a idade cronológica. Quando forma utilizados os métodos baseados em Atlas, os valores da média da diferença e os desvios padrão correspondentes, foram maiores que os encontrados com o TW3-RUS. Ainda que para todos os métodos a média da diferença total IO-IC não superou o ano, a precisão do TW3-RUS foi melhor, porém, no diagnóstico da idade, devem utilizar-se todos os indicadores de idade disponíveis para possibilitar uma avaliação mais precisa; assim como os fatores de correção calculados para a melhor adaptação dos métodos a amostra estudada.

\section{Referências}

1. Tanner JM, Whitehouse RH, Cameron N, Marshall WA, Healy MJR, Goldstein NH. Assessment of skeletal maturity and prediction of adult height (TW3 method). $3^{\text {rd }}$ ed. London: WB Saunders, 2001.

2. Martin DD, Wit JM, Hochberg Z, Sävendahl L, van Rijn RR, Fricke O, Cameron N, Caliebe J, Hertel T, Kiepe D, AlbertssonWikland K, Thodberg HH, Binder G, Ranke MB: The use of boné age in clinical practice-part I. Horm Res Paediatr. 2011; 76: 1-9.

3. Study Group of Forensic Age Estimation of the German Association of Forensic Medicine. 2001. Guidelines for Age
Estimation in Living Individuals in Criminal Proceedings [internet]. 2001. [cited 2013 Feb 28]. Available from: agfad.unimuenster.de/English/empfehlungen.htm.

4. Schmeling A, Garamendi PM, Prieto JL, Landa MI. Forensic age estimation in unaccompanied minors and young living adults In: Vieira DN, editor [internet]. Intech ; 2011. [cited 2013 Feb 28]. Available from: http: //www. Interchopen.com/ books/forensic-medicine-from-old-problems-to newchaleenges/forensic-age-estimation-in-unaccompanied-minors-and-young-living-adults.

5. Greulich WW, Pyle SI. Radiographic atlas of skeletal development of the hand and wrist. $2^{\text {nd }}$. ed. Stanford: Stanford University Press, 1959.

6. Fundación Centro de Estudio sobre Crecimiento y Desarrollo de la Población Venezolana. Atlas de maduración ósea del venezolano. Caracas: Ministerio de Salud y Desarrollo Social, 2003.

7. Roche AF, Davila GH, Eyman SL. A comparison between Greulich-Pyle and Tanner-Whitehouse assessment of skeletal maturity. Radiology. 1971; 98: 273-80.

8. Moraes LC, Moraes MEL. Verifição da assimetria bilateral de desenvolvimento por meio de radiografias de mão e punho, baseada na avaliação da idade óssea. Rev Odontol UNESP. 1996; 25: 183-94.

9. Carvalho AAF. Estimativa de valores médios de crescimento em crianças com idade cronológica variando de 84 a 131 meses. Rev Odontol UNESP. 1993; 22: 293-301.

10. Haiter-Neto F, Almeida SM, Leite CC. Estudo comparativo dos métodos de estimativa da idade óssea de Greulich \& Pyle e Tanner \& Whiteouse. Pesqui Odontol Bras. 2000; 14: 378-84.

11. Haavikko K, Kilpinen E. Skeletal development of Finnish children in the light of hand-wrist roentgenograms. Proc Finn Dent Soc. 1973; 69: 182-90.

12. Patil ST, Parchand MP, Meshram MM; Kamdi NY. Applicability of Greulich and Pyle skeletal age standards to Indian children. Forensic Sci Int. 2012; 216: e1-4.

13. Vignolo M, Milani S, Di battista E, Naselli A, Mostert M, Aicardi G. Modified Greulich-Pyle, Tanner-Whitehouse, and RocheWainer-Thissen (knee) methods for skeletal age assessment in a group of Italian children and adolescents. Eur J Pediatr. 1990; 149: 314-7.

14. Groell R, Lindbichler F, Riepl T, Gherra L, Roposch A, Fotter $R$. The reliability of bone age determination in central European children using the Greulich and Pyle method. Br J Radiol. 1999; 72: 461-4.

15. Lewis CP, Lavy CBD, Harrison WJ. Delay in skeletal maturity in Malawian children. J Bone Joint Surg Br. 2002; 84: 732-4.

16. Koc A, Karaoglanoglu M, Erdogan M, Kosecik M, Cesur Y. Assessment of bone ages: Is the Greulich-Pyle method sufficient for Turkish boys? Pediatr Int. 2001; 43: 662-5.

17. Lejarraga H, Guimarey L, Orazi V. Skeletal maturity of the hand and wrist of healthy Argentinean children aged 4-12 years, assessed by the TW II method. Ann Hum Biol. 1997; 24: $257-61$.

18. Vignolo M, Naselli A, Magliano P, Di Battista E, Aicardi M, Aicardi G. Use of the new US90 standards for TW-RUS skeletal maturity scores in youths from the Italian population. Horm Res. 1999; 51:168-72.

19. Ashizawa K, Asami T, Anzo M, Matsuo N, Matsuoka H, Murata M, Ohtsuki F, Satoh M, Tanaka T, Tatara H, Tsukagoshi K. Standard RUS skeletal maturation of Tokyo children. Ann Hum Biol. 1996; 23:457-69. 
20. Ortega Al, Haiter-Neto F, Ambrosano GMB, Bóscolo FN Almeida SM, Casanova MS. Comparison of TW2 and TW3 skeletal age differences in a Brazilian population. J Appl Oral Sci. 2006; 14: 142-6.

21. Amaral TPM, Barros Jr VN, Barroso AB, Haiter-Neto F, Pordeus IA, Silva Freire AR. Estimativa da idade óssea das crianças brasileiras utilizando o atlas de Greulich \& Pyle. In: Sociedade Brasileira de Pesquisa Odontológica, editor. 19a Reunião Anual da Sociedade Brasileira de Pesquisa Odontológica: Pesqui Odontol Bras. 16(supl. 1); 2002 Agos 31-Set 4; Aguas de Lindoia (SP), São Paulo; 2002. p. 254.

22. Moraes MEL, Moraes LC, Medici Filho E, Graziosi MAOC. Fidelidade dos métodos Greulich \& Pyle e Eklöf \& Ringertz para avaliação da idade óssea em crianças brasileiras. Rev Odontol UNESP. 2003; 32: 9-17.

23. Zhang A, Sayre JW, Vachon L, Liu BJ, Huang HK: Racial differences in growth patters of children assessed on the basis of bone age. Radiology. 2009; 250: 228-35.

24. Calfee RP, Sutter M, Steffen JA, Goldfarb CA. Skeletal and chronological ages in American adolescents: current findings in skeletal maturation. J Child Orthop. 2010; 4: 467-70.
25. Büken B, aafak AA, Büken E, Yazigi B, Erkol Z, Erzengin ÖU. Is the Tanner-Whitehouse (TW3) method sufficiently reliable for forensic age determination of Turkish children? Turk J Med Sci. 2010; 40: 797-805.

26. Cantekin K, Celikoglu M, Miloglu O, Dane A, Erdem A. Bone age assessment: the applicability of the Greulich-Pyle method in eastern Turkish children. J Forensic Sci. 2012; 57: 679-82.

27. Rikhasor R, Qureshi AM, Rathi SL, Chana NA. Skeletal maturity in Pakistani children. J Anat. 1999; 195: 305-8.

28. Haiter-Neto F, Kurita LM, Menezes AV, Casanova MS. Skeletal age assessment: a comparison of 3 methods. Am J Orthod Dentofacial Orthop. 2006; 130: 435.e15-20.

29. Loder RT, Estle DT, Morrison K, Eggleston D, Fish DN; Greenfield ML, Guire KE. Applicability of the Greulich and Pyle skeletal age standards to black and white children of today. Am J Dis Child. 1993; 147: 1329-33.

30. Schmidt S, Nitz I, Schulz R, Schmeling A. Applicability of the skeletal age determination method of Tanner and Whitehouse for forensic diagnostics. Int J Legal Med 2008; 122: 309-14. 